Изв. Крымск. Астрофиз. Обсерв. 116, № 2, 18-33 (2020) doi:10.31059/izcrao-vol116-iss2-pp18-33

\title{
Звезды типа Т Тельца: магнитные поля и планеты
}

\author{
К.Н. Гранкин и сотрудничество MaTYSSE \\ ФГБУН “Крымская астрофизическая обсерватория РАН”, Научный, Крым, 298409 \\ konstantin.grankin@craocrimea.ru
}

Поступила в редакцию 21 августа 2020 г.

Аннотация. В этом кратком обзоре мы представляем результаты исследования крупномасштабных магнитных полей у звезд типа Т Тельца (TTS). Небольшой спектрополяриметрический обзор 8 молодых звезд был осуществлен в рамках двух международных проектов МаРP (Magnetic Protostars and Planets) и MaTYSSE (Magnetic Topologies of Young Stars and the Survival of massive close-in Exoplanets) в период с 2009 по 2016 годы. Для каждого нашего объекта мы реконструировали карту яркости и топологию магнитного поля с использованием метода доплер-зеемановского картирования (ZDI). Обзор содержит краткое описание спектрополяриметрических данных, метода ZDI, пример восстановления яркостных и магнитных карт, а также свойства магнитных полей 8 TTS. Hаши результаты показывают, что AA Tau и LkCa 15 взаимодействуют со своими дисками в режиме пропеллера, когда звездное вращение активно замедляется магнитным взаимодействием между звездой и диском. Мы обнаружили, что магнитные поля некоторых TTS являются переменными на временном масштабе в несколько лет и, следовательно, по своей природе нестационарны. Мы сообщаем об открытии двух гигантских экзопланет вокруг V830 Tau и TAP 26. Эти два новых обнаружения предполагают, что теория дисковой миграции 2-го типа хорошо объясняет образование горячих юпитеров около молодых TTS. Результаты нашего обзора сравниваются с общей картиной свойств магнитных полей двадцати TTS на диаграмме Герцшпрунга - Рассела. Сравнение показывает, что WTTS демонстрируют более широкий диапазон различных топологий магнитного поля по сравнению с CTTS, и что магнитное поле TTS (CTTS и WTTS) в основном полоидальное и осесимметричное у более холодных (меньше 4300 K) звезд, которые остаются в значительной степени конвективными. Эти выводы нуждаются в подтверждении на основании анализа более представительной выборки молодых звезд.

Ключевые слова: молодые звезды, магнитные поля, протопланетные диски, экзопланеты

\section{1 Введение}

Звезды типа T Тельца (T Tauri stars, TTS) представляют собой молодые маломассивные звезды поздних спектральных классов, которые находятся на стадии гравитационного сжатия и еще не достигли главной последовательности (ГП). Некоторые из этих объектов, “классические” TTS (classical TTS, CTTS), окружены достаточно массивными аккреционными газопылевыми протопланетными дисками. Другие TTS со слабыми эмиссионными линиями (weak-line TTS, WTTS) не показывают признаков наличия каких-либо дисков вообще или имеют так называемые "переходные" диски, которые потеряли пылевую составляющую во внутренних областях в силу процессов аккреции и формирования планетных систем.

Наличие магнитных полей у TTS в значительной степени определяет характер взаимодействия между звездой и ее аккреционным диском, играет решающую роль в эволюции углового момента сжимающейся звезды и может влиять на формирование и миграцию молодых планет. Таким образом, изучение свойств магнитных полей TTS имеет первостепенное значение для тестирования и развития моделей звездного динамо и теорий формирования звезд солнечного типа и их планет. С целью изучения крупномасштабной топологии магнитных полей TTS были организованы две международные исследовательские программы MaPP (Magnetic Protostars and Planets) и MaTYSSE 
(Magnetic Topologies of Young Stars and the Survival of massive close-in Exoplanets). Первая программа была реализована в 2009-2012 гг., вторая - в 2013-2016 гг.

Эти две исследовательские программы объединили усилия ученых из Франции, Великобритании, Канады, США, Тайваня, Бразилии, Чили, Португалии, Китая, Италии, Швейцарии и астрономов из Крымской астрофизической обсерватории. Большая часть наблюдений, выполненных в рамках программы МаРP, была получена с использованием спектрополяриметра ESPaDOnS на 3.6-м телескопе CFHT (Гавайи). Во время реализации второй программы МаTYSSE использовались дополнительно еще два спектрополяриметра: NARVAL на 2-м телескопе TBL (Пик-дю-Миди, Франция) и HARPSPol на 3.6-м телескопе ESO (Ла-Силла, Чили). В данном обзоре представлены наиболее интересные результаты из программ MaPP и MaTYSSE, которые были получены при непосредственном участии исследователей из КрАО.

\section{2 Спектрополяриметрические данные}

Подавляющее большинство спектральных данных избранных молодых звезд было получено в течение одного или двух наблюдательных сезонов. Каждый наблюдательный сет имел продолжительность от одной до двух недель, и спектральные данные покрывали от 5 до 10 вращательных циклов звезды. Как правило, каждый спектр охватывал весь оптический диапазон (от 370 до 1000 нм) с разрешением 65000 (т. е. 4.6 км с ${ }^{-1}$ ) и спектральной дискретизацией $2.6 \mathrm{~km} \mathrm{c}^{-1}$. Для каждого объекта были получены как неполяризованные (I-параметр Стокса), так и поляризованные спектры ( $V$-параметр Стокса). Все поляризованные спектры состоят из четырех отдельных подэкспозиций, полученных в разных положениях поляриметра, что позволяет учитывать инструментальные поляризационные эффекты первого порядка.

Все исходные спектры обрабатывались с помощью специализированного пакета Libre Esprit, доступного на телескопах CFHT и TBL. Этот пакет автоматически выполняет оптимальное извлечение неполяризованных и поляризованных спектров. Благодаря тому, что щель спектрографа имеет наклон относительно строк ПЗС, спектральные порядки, соответствующие различным строкам ПЗС, имеют различную выборку пикселей. Пакет Libre Esprit использует эту возможность для оптимального извлечения спектров с более плотной дискретизацией, чем исходная дискретизация самой ПЗС. В результате достигается спектральное разрешение около $1.8 \mathrm{~km} \mathrm{c}^{-1}$. Все спектры автоматически корректируются по спектральным сдвигам, возникающим в результате инструментальных ошибок (например, из-за механических изгибов, из-за колебаний температуры или давления). Для этого в качестве эталона используются теллурические линии. Хотя эти процедуры пакета Libre Esprit и не идеальны, они обеспечивают точность определения лучевой скорости (RV) лучше $30 \mathrm{~m} \mathrm{c}^{-1}$.

Таблица 1. Параметры звезд и спектральных данных

\begin{tabular}{llrrlccccccc}
\hline Звезда & тип & $\begin{array}{c}T_{\text {eff }} \\
\mathrm{K}\end{array}$ & $\begin{array}{r}v \sin i \\
\text { км с }^{-1}\end{array}$ & $\begin{array}{c}P_{\text {rot }} \\
\text { дни }\end{array}$ & $\begin{array}{c}\mathrm{M} \\
M_{\odot}\end{array}$ & $\begin{array}{c}\mathrm{t} \\
\text { млн л }\end{array}$ & $\begin{array}{c}\text { эпоха } \\
2000+\end{array}$ & $N_{s}$ & $N_{\text {obs }}$ & $S / N$ & $\overline{S / N}$ \\
\hline AA Tau & $\mathrm{c}$ & 4000 & 11.3 & 8.22 & 0.70 & 1.5 & $07-09$ & 2 & 24 & $100-200$ & 150 \\
V2129 Oph & $\mathrm{c}$ & 4500 & 14.5 & 6.53 & 1.35 & $2-3$ & 09 & 1 & 23 & $100-210$ & 180 \\
LkCa 4 & $\mathrm{w}$ & 4100 & 28.0 & 3.374 & 0.79 & 2 & 14 & 1 & 12 & $120-180$ & 160 \\
LkCa 15 & $\mathrm{c}$ & 4500 & 13.0 & 5.70 & 1.25 & 5 & 15 & 1 & 14 & $110-180$ & 160 \\
TAP 26 & $\mathrm{w}$ & 4620 & 68.2 & 0.7135 & 1.04 & 17 & $15-16$ & 2 & 29 & $100-150$ & 140 \\
V819 Tau & $\mathrm{w}$ & 4250 & 9.5 & 5.5311 & 1.00 & 3.2 & $14-15$ & 1 & 30 & $140-220$ & 190 \\
V830 Tau & $\mathrm{w}$ & 4250 & 30.5 & 2.7410 & 1.00 & 2.2 & $14-16$ & 2 & 56 & $80-170$ & 150 \\
V410 Tau & $\mathrm{w}$ & 4500 & 73.2 & 1.87197 & 1.42 & 0.8 & $08-16$ & 4 & 129 & $82-238$ & 140 \\
\hline
\end{tabular}

Пиковые отношения сигнал/шум, достигнутые в полученных спектрах, находятся в диапазоне от 70 до 90 для данных NARVAL и от 100 до 220 для данных ESPaDOnS. Основная информация о звездах и полученных спектральных данных собрана в таблице 1, где приведены имя звезды, тип (“с", если CTTS, или "w", если WTTS), эффективная температура, значение $v \sin i$, период вращения, масса, возраст, эпоха спектральных наблюдений, количество наблюдательных сезонов $\left(N_{s}\right)$, количество спектров, диапазон значений отношения сигнал/шум $(S / N)$ и среднее значение отношения сигнал/шум $(\overline{S / N})$. 


\section{3 Метод доплер-зеемановского картирования}

Для анализа спектральных данных была использована техника деконволюции по методу наименьших квадратов (Least-Squares Deconvolution, LSD). Эта техника взаимной корреляции позволяет вычислять средний профиль одной псевдолинии из нескольких тысяч спектральных линий, которые ведут себя одинаковым образом в магнитном поле. Основное преимущество методики LSD состоит в существенном повышении результирующего отношения $\mathrm{S} / \mathrm{N}$ при построении как $V$-, так и $I$-профиля псевдолинии. Список линий, которые использовались для вычисления среднего локального профиля LSD, формировался на основе модели атмосферы в условиях локального термодинамического равновесия ATLAS9 для определенного спектрального класса и значения $\log g$, которые соответствуют конкретной исследуемой звезде. В этот список включались только умеренные или сильные атомные спектральные линии, формирующиеся в фотосфере звезды. Как правило, при вычислении среднего локального профиля LSD использовалось от семи до девяти тысяч спектральных линий, причем около 40 \% из них - это линии Fe I. B результате отношение S/N для среднего LSD I-профиля достигало значений от 950 до 3000 для скоростного бина $1.8 \mathrm{kм} \mathrm{c}^{-1}$. Отношение $\mathrm{S} / \mathrm{N}$ для LSD $V$-профиля, где преобладает шум статистики фотонов, варьировалось в диапазоне от 1800 до 7000 в зависимости от яркости объекта и погодных условий.

Чтобы восстановить распределение яркости по поверхности звезды и реконструировать топологию магнитного поля, мы использовали томографический метод доплер-зеемановского картирования (Zeeman-Doppler imaging, ZDI). Этот метод позволяет преобразовывать одновременные временные ряды одномерных $I$ - и $V$-профилей LSD в двумерные изображения: карту поверхностной яркости (яркостную карту) и карту магнитного поля (магнитную карту). С математической точки зрения ZDI следует принципам восстановления изображения методом максимальной энтропии и итеративно ищет изображение с наименьшим информационным содержанием, которое соответствует некоторому заданному уровню $\chi^{2}$. Поверхностное магнитное поле моделируется как комбинация полоидального и тороидального полей, представленных в виде взвешенных сумм сферических гармоник (с порядками от $l=1$ до $l=15$ ), которые проецируются на сферическое координатное пространство (Donati et al., 2010).

В модифицированной версии метода ZDI восстановленная яркостная карта позволяет выявлять не только темные пятна, но и яркие площадки. Это достигается за счет того, что восстановленная поверхностная яркость (нормализованная относительно яркости фотосферы) теперь может изменяться в пределах как ниже, так и выше единицы, а не ограничивается интервалом $[0,1]$, как это было ранее. Следует отметить, что обнаруживаемые яркие площадки относятся к области фотосферы, более яркой, чем спокойная фотосфера, а не к яркой области на уровне хромосферы, как в случае Солнца (Donati et al., 2014).

Чтобы смоделировать локальные $I$ - и $V$-профили LSD, мы использовали уравнения УнноРачковского для переноса поляризованного излучения в модельной атмосфере Милна-Эддингтона с учетом локальной яркости и магнитного поля. Затем эти локальные профили интегрировались по видимому полушарию звезды, чтобы получить синтетические средние фотосферные LSD-профили для их дальнейшего сравнения с нашими спектральными наблюдениями. Такой метод вычислений обеспечивает, в частности, надежное описание того, как профили линий искажаются в присутствии магнитных полей. Основные параметры локального LSD-профиля, использованные в методе ZDI, были следующими: центральная длина волны псевдолинии 670 нм, доплеровская ширина линии $1.8 \mathrm{kм} \mathrm{c}^{-1}$, эквивалентная ширина $3.9 \mathrm{kм} \mathrm{с}^{-1}$ и множитель Ланде 1.2.

Вычисляя величину широтного сдвига, которому подвержены яркостные карты в течение времени, метод ZDI позволяет получить оценку дифференциального вращения на фотосферном уровне. Учет дифференциального вращения способствует более чистой фильтрации исходных кривых RV и позволяет проводить поиск сигнала от планеты в остаточных кривых RV. Для получения абсолютных оценок вуалирования в случае анализа спектров CTTS использовалась звезда-стандарт с аналогичными температурой и классом светимости (как правило, WTTS), которая наблюдалась на том же спектрополяриметре. Спектр звезды-стандарта искусственно расширяли до того же значения $v \sin i$, вуалировали и выполняли кросс-корреляцию с каждым спектром исследуемой CTTS до достижения наилучшего соответствия. Эта процедура позволила одновременно учесть вуалирование и определить лучевую скорость (Alencar et al., 2018). 


\section{4 Реконструкция яркостных и магнитных карт}

В качестве примера на рис. 1 представлен набор $I$ - и $V$-профилей LSD, полученный нами для LkCa 4 в течение января 2014 года, вместе с модельной подгонкой этих данных. Подгонка, которую мы получили, соответствует минимальному значению $\chi^{2}=1$. В самом начале процедуры подгонки значение $\chi^{2}$ было больше 50, что соответствовало нулевому магнитному полю и яркостной карте без каких-либо крупных температурных неоднородностей. Из рис. 1 видно высокое качество как самого набора спектральных данных, так и результирующей подгонки. Учитывая этот факт, большое значение $v \sin i=28.0 \pm 0.5 \mathrm{kм}^{-1}$ и достаточно плотный фазовый охват, можно с уверенностью утверждать, что на восстановленных картах не следует ожидать каких-либо значительных случайных артефактов или смещений.
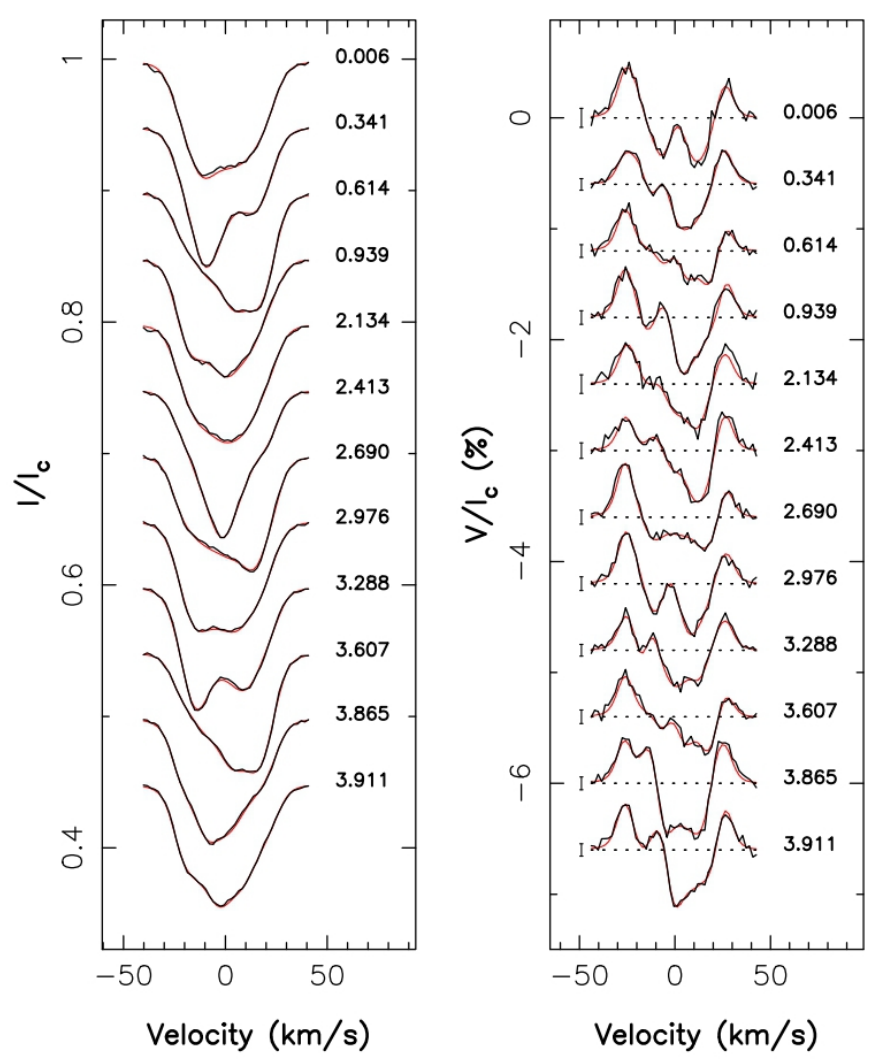

Рис. 1. Набор профилей LSD для LkCa 4, полученный в январе 2014 года. Неполяризованные ( $I$-параметр Стокса) и поляризованные ( $V$-параметр Стокса) профили LSD показаны на левой и правой панели соответственно. Наблюденные профили обозначены толстой черной линией, результаты подгонки методом максимальной энтропии - тонкой красной линией. Вращательные циклы и бары ошибок (для $V$-профиля Стокса) показаны рядом с каждым профилем (рис. 3 из работы Donati et al., 2014)

На рис. 2 приведены результирующие карты, которые мы реконструировали из набора наблюденных $I$ - и $V$-профилей с использованием метода ZDI. Главной особенностью яркостной карты (верхняя левая панель на рис. 2) является темное пятно сложной формы вблизи полярной области. Как оказалось, это пятно неосесимметричное, с несколькими придатками, которые опускаются к более низким широтам, о чем свидетельствует значительная временная изменчивость в неполяризованных профилях LSD (левая панель на рис. 1). Второй наиболее заметной особенностью яркостной карты является наличие яркой площадки, расположенной на средних широтах вблизи фазы 0.42 . Яркость этой площадки на 50 \% выше, чем средняя яркость окружающей спокойной фотосферы. Темное пятно и яркая область покрывают в общей сложности $25 \%$ всей поверхности звезды. Следует отметить, что фотометрические изменения блеска, предсказанные реконструированной яркостной картой, находятся в очень хорошем соответствии с фотометрическими наблюдениями, полученными 
в KрАО в течение четырех месяцев. Это говорит еще и о том, что время жизни яркостных элементов на поверхности LkCa 4 превышает несколько месяцев, что полностью совместимо с предыдущими выводами долгосрочного фотометрического мониторинга, показывающими, что кривые блеска этой звезды удивительно стабильны во времени как по форме, так и по фазе (см. рис. 5 в работе Grankin et al. (2008)).
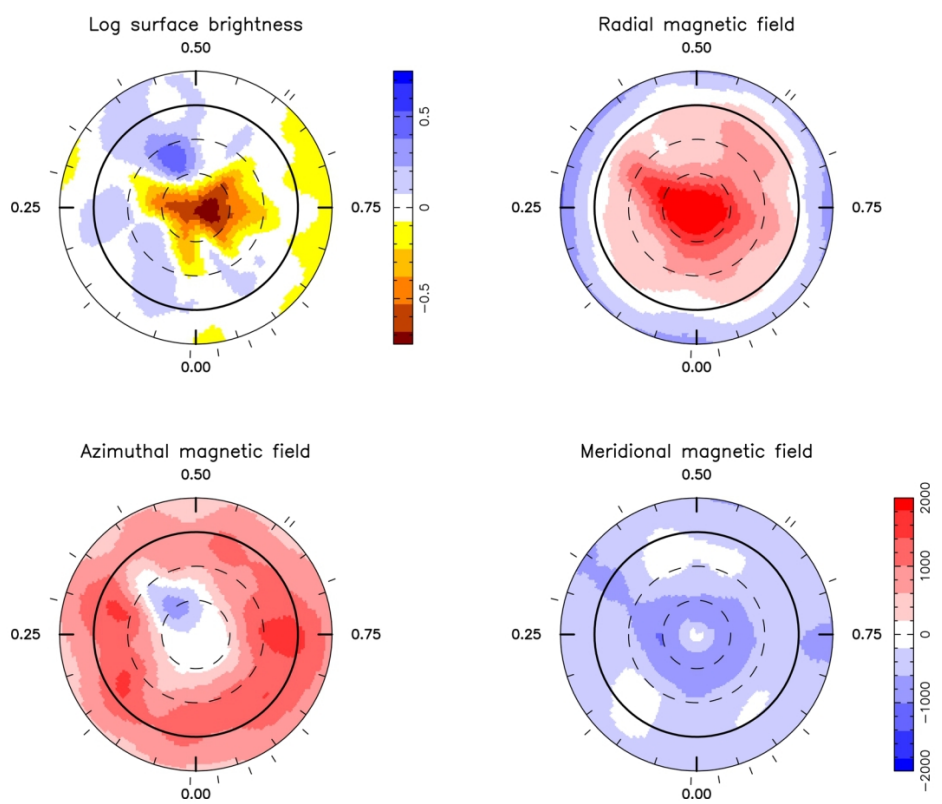

Рис. 2. Карты логарифмической яркости (относительно спокойной фотосферы, верхняя левая панель), радиальной (верхняя правая панель), азимутальной (нижняя левая панель) и меридиональной (нижняя правая панель) составляющих магнитного поля на поверхности LkCa 4 в январе 2014 года. Магнитные потоки показаны цветом от -2000 до 2000 Гс. На всех панелях звездная поверхность представлена в плоской полярной проекции до широты $-30^{\circ}$. Экватор изображен жирным кругом, а параллели - пунктирными кругами. Радиальные штрихи вокруг каждого изображения указывают фазы спектральных наблюдений (рис. 4 из работы Donati et al., 2014)

Магнитная карта демонстрирует довольно простую крупномасштабную магнитную структуру, состоящую из двух основных компонентов. Первый компонент - это осесимметричное полоидальное поле, содержащее в себе до $70 \%$ магнитной энергии. При этом $94 \%$ энергии полоидального поля сосредоточено в модах сферических гармоник (SH) с $m<l / 2(l$ и $m$ обозначают, соответственно, степени и порядки мод), и 86 \% этой энергии содержится в дипольном компоненте $(l=1$ и $m=0)$. Полоидальный компонент может быть аппроксимирован доминирующим диполем $(1.6$ кГс), наклоненным под углом $\simeq 10^{\circ}$ к оси вращения (в сторону фазы 0.75$)$, и в 4 раза более слабым $(\simeq 0.4$ кГс) осесимметричным октупольным компонентом. В результате генерируется интенсивное радиальное поле, которое превышает 2 кГс на видимом полюсе звезды (см. верхнюю правую панель на рис. 2), которое совпадает с темным пятном, восстановленным на яркостной карте. Отметим также, что вытянутая форма этого магнитного полюса похожа на форму темного полярного пятна.

Вторым основным компонентом крупномасштабного поля этой звезды является очень значительный, почти осесимметричный тороидальный компонент, проявляющийся как кольцо азимутального поля $(\simeq 1 \mathrm{k} \Gamma$ c), которое окружает звезду на экваториальных широтах (см. нижнюю левую панель на рис. 2). Эта особенность непосредственно отражает почти симметричную форму $V$-профилей LSD (около центра профиля линии) на большинстве фаз вращения (см. правую панель на рис. 1), которая, как известно, является четким признаком заметного кольца азимутального поля на поверхности звезды (Donati et al., 2014). Мы также отмечаем обнаружение локальной особенности азимутального поля противоположной полярности (по часовой стрелке) в непосредственной близости от яркой площадки, обнаруженной на яркостной карте. Сферические гармоники, описывающие восстановленное поле, ограничены членами с $l \leq 10$. Только незначительные изменения в решении наблюдаются при 
увеличении максимального значения $l$ с 10 до 15, что свидетельствует о том, что бо́льшая часть сигнала, обнаруженного в $V$-профилях LSD LkCa 4, концентрируется на бо́льших пространственных масштабах.

\section{5 Результаты доплер-зеемановского картирования}

На рис. 3 и рис. 4 представлены яркостные и магнитные карты для 3 CTTS и 3 WTTS, восстановленные на основе спектрополяриметрических данных, полученных в рамках двух международных проектов MaPP и MaTYSSE при непосредственном участии исследователей из КрАО.

AA Tau. На яркостной карте реконструируется протяженная запятненная область сложной конфигурации, которая распространяется почти на $180^{\circ}$ в долготном направлении (рис. 3 , левая верхняя панель). Наиболее темная зона этой области находится на широте около 50 и почти совпадает с самой интенсивной областью радиальной составляющей магнитного поля. Магнитное поле содержит значительную осесимметричную полоидальную составляющую, в которой сосредоточено 80-85 \% магнитной энергии на поверхности звезды. В свою очередь, около 90-95\% энергии полоидальной составляющей сконцентрировано в дипольном компоненте $(l=1$ и $m=0)$. Фактически полоидальная составляющая может быть почти полностью аппроксимирована доминирующим диполем $\left(\simeq 2\right.$ кГс), наклоненным примерно на $20^{\circ}$ к оси вращения (в сторону фазы 0.20) (рис. 3 , средняя верхняя панель). Следует отметить, что октупольный компонент полоидального поля по меньшей мере в 5 раз меньше дипольного компонента. Это обстоятельство делает АА Таи очень похожей на М-карлики, чьи крупномасштабные поля также являются сильными, в основном полоидальными и осесимметричными (Morin et al., 2008). Мы также находим, что поле имеет заметную тороидальную составляющую, которая содержит около 15-20 \% всей магнитной энергии. Эта составляющая проявляется в виде фрагментов экваториального кольца отрицательного (направленного по часовой стрелке) азимутального поля напряженностью 0.5-1 кГс (рис. 3, правая верхняя панель).

Темп аккреции вещества протопланетного диска на поверхность АА Тац, оцененный нами из эмиссии в линии Не I $D_{3}$, является переменным, в диапазоне от $10^{-9.6}$ до $10^{-8.5} M_{\odot}$ год ${ }^{-1}$, и в среднем равен $10^{-9.2} M_{\odot}$ год ${ }^{-1}$. Это на порядок величины меньше, чем темп аккреции, при котором альфвеновский радиус (меньше которого диск разрушается магнитным полем звезды) равен радиусу коротации (на котором кеплеровский орбитальный период равен периоду вращения звезды), и при котором возможна аккреция. Это говорит о том, что АА Таu находится в режиме пропеллера, когда бо́льшая часть аккрецирующего вещества выбрасывается наружу, и только небольшая часть падает на поверхность звезды. Таким образом, вращение АА Таu эффективно тормозится из-за магнитной связи между звездой и протопланетным диском (Donati et al., 2010).

LkCa 15. Магнитная карта этой звезды показывает область сильного радиального поля на промежуточных широтах $\left(30-60^{\circ}\right)$, значительно вытянутую в долготном направлении и центрированную на фазу 0.4, где напряженность поля достигает 2.2 кГс. Этот участок интенсивного радиального поля перекрывается с протяженной запятненной областью, которая протянулась почти на $180^{\circ}$ в долготном направлении и опускается до экватора (и даже ниже) на фазе 0.4 (рис. 3, левая средняя панель). Мы находим, что магнитное поле имеет сильный осесимметричный полоидальный компонент, в котором содержится до 85 \% магнитной энергии, как и в случае АА Таu. Этот полоидальный компонент в основном состоит из диполя $(1.35 \mathrm{k} Г \mathrm{c})$, наклоненного под углом $\simeq 20^{\circ}$ к оси вращения (в сторону фазы 0.40), который содержит в себе $\simeq 75 \%$ энергии полоидального поля. Полоидальное поле также включает в себя октупольную составляющую противоположного знака $(-0.9$ кГс), которая собирает $\simeq 20$ \% энергии полоидального поля (Donati et al., 2019).

Средний темп аккреции вещества диска на поверхность LkCa 15 составляет $10^{-9.2} M_{\odot}$ год ${ }^{-1}$. Это означает, что сильное магнитное поле LkCa 15 способно очистить внутренние области протопланетного диска до расстояния 0.07 а.е., на котором кеплеровский орбитальный период равен периоду вращения звезды. Наши результаты показывают, что LkCa 15, так же как и AA Tau, находится в режиме пропеллера, при котором происходит эффективное замедление вращения молодой звезды сильным дипольным компонентом магнитного поля.

V2129 Oph. Реконструированная крупномасштабная топология магнитного поля V2129 Oph в июле 2009 года является почти полностью полоидальной: только 5 \% магнитной энергии содержится в тороидальном компоненте. В отличие от AA Tau и LkCa 15, полоидальное поле V2129 Oph не является осесимметричным, причем октупольная составляющая в 2-3 раза сильнее диполя. Максималь- 

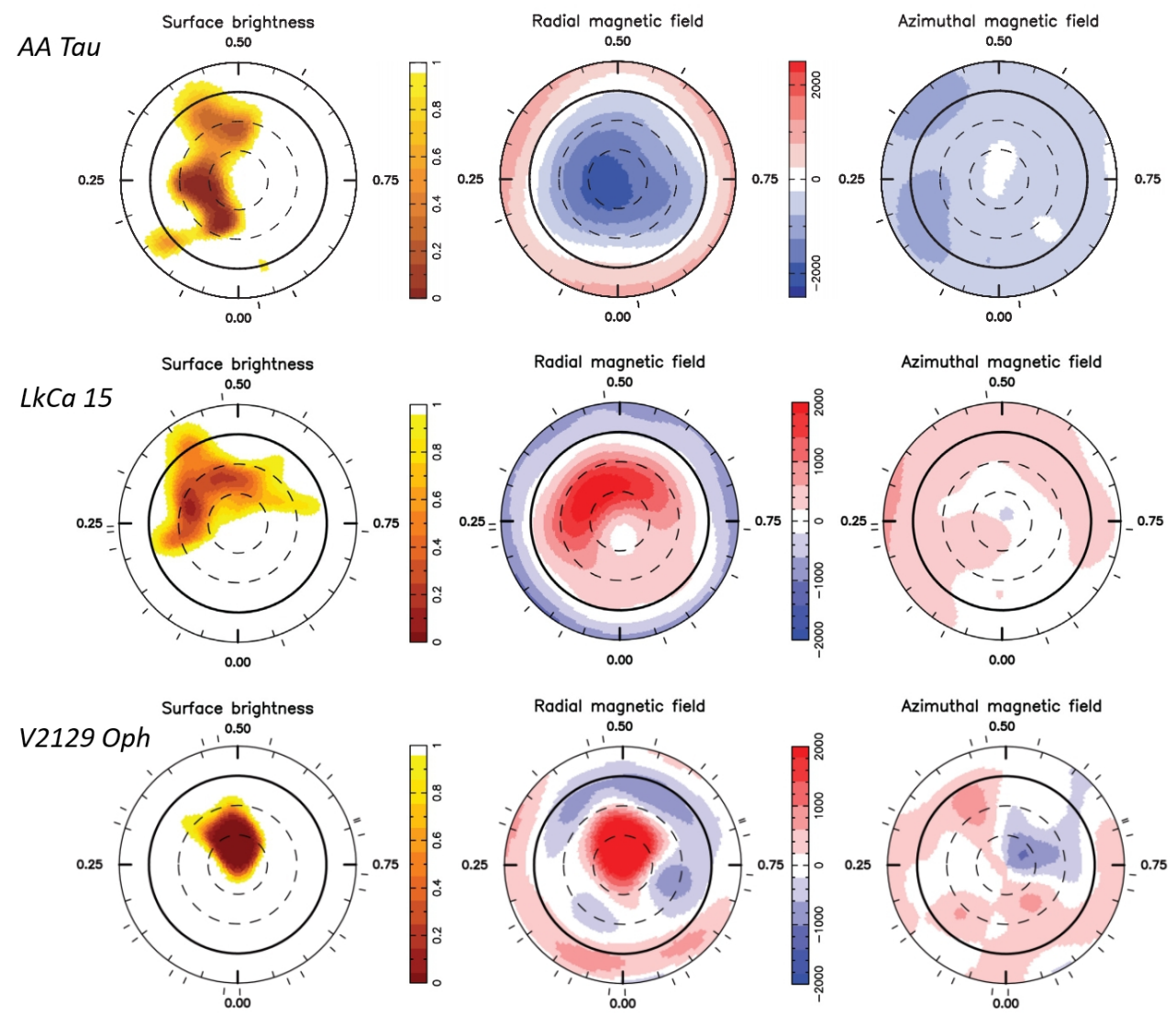

Рис. 3. Карты яркости (левая панель), радиальной (средняя панель) и азимутальной (правая панель) составляющих магнитного поля на поверхности трех CTTS: AA Tau (в конце 2007 года), LkCa 15 (в ноябре 2015 года) и V2129 Oph (в июле 2009 года). Обозначения как на рис. 2. Этот рисунок построен на основе рис. 5 из Donati et al. (2010), рис. 3 из Donati et al. (2019) и рис. 6 из Donati et al. (2011)

ная напряженность октупольной и дипольной компонент составляет около 2.1 и 0.9 кГс соответственно. Основной структурой, которую мы реконструируем, является интенсивная область радиального поля на высоких широтах, где напряженность радиального поля достигает 4 кГс (рис. 3, средняя нижняя панель). Эта область совпадает с темным пятном, реконструированным на яркостной карте (рис. 3, левая нижняя панель). Октупольный компонент полоидального поля непосредственно прослеживается как фрагментированное экваториальное кольцо отрицательного радиального поля, которое окружает основную высокоширотную область радиального поля (Donati et al., 2011).

Сравнение этой конфигурации поля, восстановленной по нашим наблюдениям 2009 года, с конфигурацией поля 2005 года, реконструированной в более ранней работе Donati et al. (2007), показало, что она может претерпевать значительные изменения. Мы обнаружили, что дипольный компонент увеличился почти в 3.5 раза (с 280 до 970 Гс) по сравнению с 2005 годом, а октупольный компонент возрос в 1.3 раза (с 1620 до 2160 Гс). При этом отношение интенсивности октупольной составляющей к дипольной уменьшилось в 2.6 раза, примерно с 5.8 (в 2005 году) до 2.2 (в 2009 году). Этот результат предполагает, что магнитные поля молодых звезд могут быть переменными на временном масштабе в несколько лет и, таким образом, являются нестационарными. Такое предположение блестяще подтвердилось в результате анализа наших многолетних спектральных наблюдений V410 Тau, о чем будет сказано ниже.

V819 Tau. Яркостная карта этой звезды (см. рис. 4, левая верхняя панель) напоминает карту $\mathrm{LkCa} 4$ (см. рис. 2, левая верхняя панель) с темным пятном вблизи полюса. Рядом с ним находится светлая площадка меньшей контрастности. Более низкое пространственное разрешение на 
поверхности V819 Tau по сравнению с LkCa 4 связано с более медленным вращением этой звезды $\left(v \sin i=9.5 \pm 0.5 \mathrm{kм} \mathrm{c}^{-1}\right)$, в случае LkCa 4 мы имеем $v \sin i=28.0 \pm 0.5 \mathrm{kм} \mathrm{c}^{-1}$. Холодное пятно охватывает $9 \%$ всей поверхности, а теплое - $6 \%$. Следует отметить, что эти оценки площади пятен надо рассматривать только как нижние пределы. Дело в том, что метод ZDI эффективен для восстановления только крупномасштабных образований, которые вызывают обнаруживаемые искажения профиля линии, и он нечувствителен к мелким структурам, которые более или менее равномерно распределены по поверхности звезды (Donati et al., 2015).

Магнитное поле намного слабее, чем у LkCa 4, и в основном полоидальное (80 \% магнитной энергии) со средним значением 370 Гс. Большая часть полоидального поля концентрируется в дипольной составляющей $(75 \%)$. В первом порядке и на некотором расстоянии от звезды полоидальное поле V819 Tau может быть аппроксимировано диполем (400 Гс), наклоненным под углом $30^{\circ}$ к оси вращения по направлению к фазе 0.65 (см. рис. 4, средняя верхняя панель). Более слабая октупольная составляющая полоидального поля (100-150 Гс) более или менее выравнена по оси вращения и противоположна по знаку дипольной составляющей. Крупномасштабное поле V819 Таu включает также слабый тороидальный компонент $(170$ Гc), топология которого более сложная и менее осесимметричная, чем у LkCa 4 (рис. 4, правая верхняя панель).

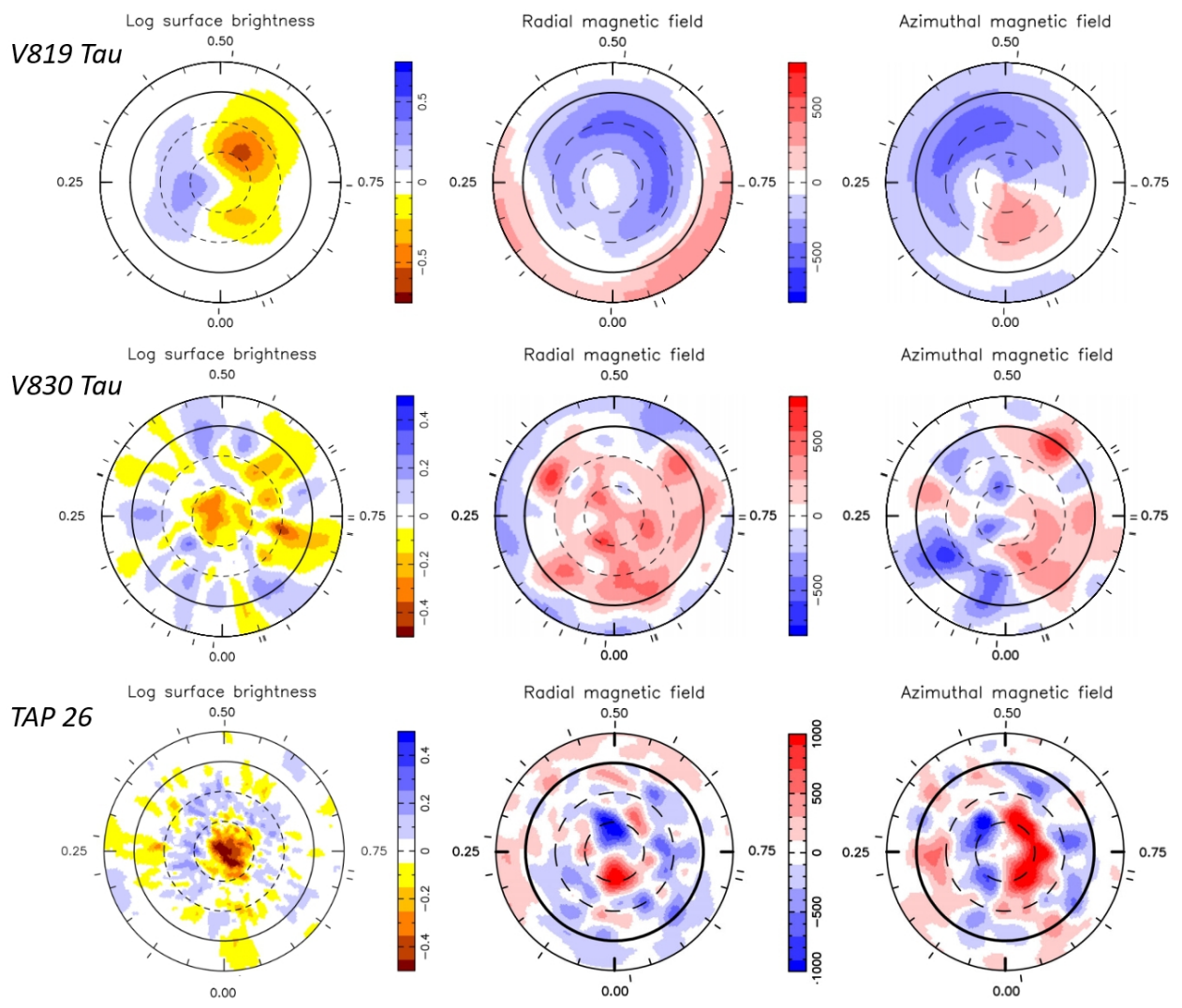

Рис. 4. Карты логарифмической яркости (левая панель), радиальной (средняя панель) и азимутальной (правая панель) составляющих магнитного поля на поверхности трех WTTS: V819 Tau (в декабре 2014 январе 2015 года), V830 Tаu (в январе - феврале 2016 года) и TAP 26 (в январе 2016 года). Обозначения как на рис. 2. Этот рисунок построен на основе рис. 4 и рис. 6 из Donati et al. (2015), рис. 3 и рис. 5 из Donati et al. (2017) и рис. 3 и рис. 5 из Yu et al. (2017)

V830 Tau. Яркостная карта V830 Таи имеет более сложную структуру по сравнению с аналогичной картой V819 Таu (рис. 4, левая средняя панель). Нижние пределы для площади холодных пятен и теплых площадок составляют $7 \%$ и $6 \%$ соответственно. Следует отметить, что помимо большого холодного полярного пятна имеется несколько протяженных областей, опускающихся до экватора и ниже (Donati et al., 2017). 
Магнитное поле V830 Tau в основном полоидальное (90 \% магнитной энергии) со средним значением 350 Гс. Большая часть полоидального поля концентрируется в дипольной составляющей (60\%). В первом порядке и на некотором расстоянии от звезды полоидальное поле V830 Таu может быть аппроксимировано диполем (340 Гс), наклоненным под углом $22^{\circ}$ к оси вращения по направлению к фазе 0.88. Октупольный компонент полоидального поля слабый $(150$ Гс) и неосесимметричный. Крупномасштабное поле V830 Таu включает также слабый тороидальный компонент (110 Гс), топология которого более сложная, чем у LkCa 4 и V819 Tau (рис. 4, правая средняя панель).

TAP 26. Яркостная карта ТАР 26, восстановленная по нашим январским спектральным наблюдениям 2016 года, приведена на рис. 4 на левой нижней панели. Помимо наиболее холодного пятна, расположенного в полярной области, на поверхности звезды присутствует большое число мелких темных пятен, которые чередуются с подобными теплыми площадками. Среди темных низкоширотных образований можно выделить две низкоширотные запятненные области, расположенные на фазах 0.27 и 0.97. Холодные пятна охватывают не менее $7 \%$ всей поверхности, а теплые - не менее 5 \%. Средняя напряженность крупномасштабного магнитного поля около 430 Гс. Поле в основном полоидальное (до 70 \% магнитной энергии), хотя и имеет значительную тороидальную составляющую (до 30 \%). Поле в значительной степени осесимметричное, особенно это касается тороидального компонента (на 80 \%). Полоидальный компонент является осесимметричным только на 50 \% (Yu et al., 2017). Дипольная компонента (120 Гс) концентрирует в себе только $10 \%$ энергии полоидального поля, в то время как октупольные и квадрупольные моды собирают до 90 \% энергии полоидального поля.

\section{6 Нестационарные магнитные поля}

Среди звезд нашей выборки V410 Таu представляет особый интерес в связи с особенностями ее фотометрического поведения. Почти два десятилетия наших фотометрических наблюдений эта звезда демонстрировала очень стабильную фазовую кривую блеска. В течение 1986-2004 гг. изменения фазы минимума блеска не превысили $0.16 P_{\text {rot }}$, где $P_{\text {rot }}-$ период вращения (см. рис. 3 в работе Grankin et al., 2008). При этом амплитуда кривой блеска изменялась в пределах от $0 .^{m} 25$ до $0 .^{m} 63$ в полосе $V$. Подобное фотометрическое поведение характерно для нескольких наиболее активных и молодых WTTS, в том числе и для LkCa 4 (Grankin, 2013). Однако в 2004-2006 годах фазовая кривая блеска начала эволюционировать и около фазы 0.9 появился второй небольшой максимум. В 2007 году амплитуда переменности достигла рекордного минимума $\left(0 .^{m} 05\right)$ при почти неизменном среднем уровне блеска (см. рис. 2 в работе Grankin, Artemenko, 2009). Согласно нашей фотометрической модели, почти полное исчезновение амплитуды периодического процесса в 2007 году было связано с тем, что темные пятна перестали концентрироваться на определенной долготе и почти равномерно распределились по звездной поверхности. Поскольку изменение распределения пятен по поверхности звезды отражает изменения в структуре магнитного поля, есть веские основания говорить о том, что топология крупномасштабного магнитного поля V410 Таu претерпела серьезные изменения в 2006-2008 годах. Первые спектрополяриметрические наблюдения этой звезды, предпринятые нами в 2008 году, показали, что на поверхности звезды присутствует как полярное пятно, так и несколько низкоширотных пятен, которые распределены в долготном направлении довольно равномерно (Skelly et al., 2010), что мы и предсказывали в более ранних работах.

Чтобы исследовать магнитное поле V410 Таu и проследить возможную эволюцию его основных параметров с течением времени, мы предприняли долговременные спектрополяриметрические наблюдения этой звезды. Наш набор спектрополяриметрических данных охватывает период времени с октября 2008 г. по январь 2016 г. (см. последнюю строку в таблице 1). Полный журнал наблюдений представлен в нашей работе Yu et al. (2019). Результаты реконструкции яркостных и магнитных карт, полученные в течение шести сезонов, приведены на рис. 5. Яркостные карты демонстрируют сложную структуру с большим числом относительно мелких и контрастных деталей. Для всех карт характерна большая концентрация темных пятен в районе полюса. В январе 2009 г., декабре 2013 г. и декабре 2015 г. на картах присутствует большое контрастное темное пятно в области экватора, расположенное на фазах $0.27,0.48$ и 0.48 соответственно. Остатки этого пятна (запятненной области) наблюдаются и на карте, соответствующей январю 2016 г., где его интенсивность стала меньше. Менее контрастная, но более протяженная и сложная по форме запятненная экваториальная область прослеживается в диапазоне фаз 0.6-0.7 в январе 2009 г. В январе 2011 г. она распадается на 
Log surface brightness Radial field

Meridional field

Azimuthal field
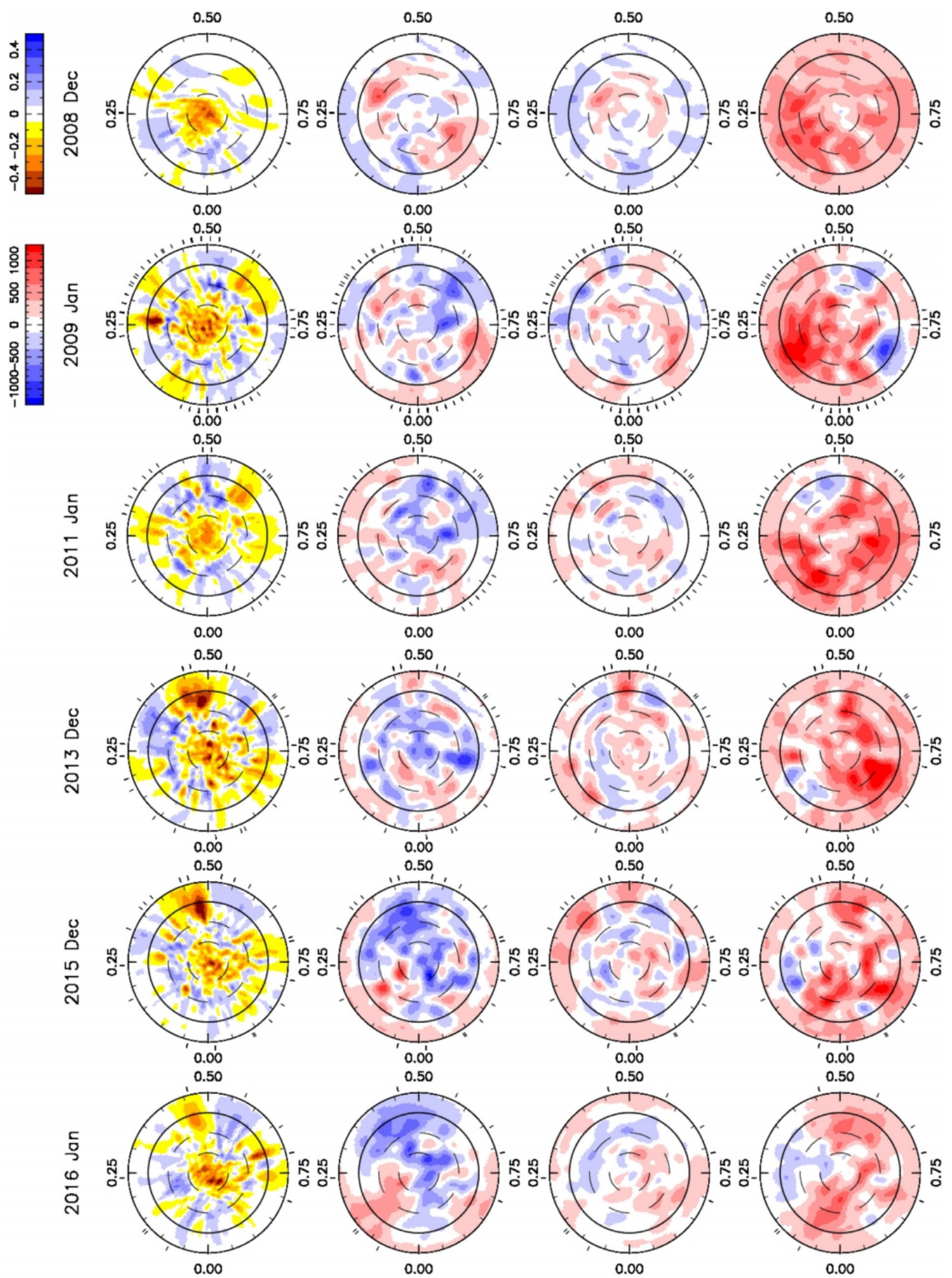

Рис. 5. Карты логарифма относительной яркости (правый столбец) и радиальной, меридиональной и азимутальной компонент магнитного поля (второй-четвертый столбцы) на поверхности V410 Tau, восстановленные по данным, полученным в 2008-2016 гг. (строки сверху вниз). Обозначения как на рис. 2. (рис. 3 из работы Yu et al., 2019) 

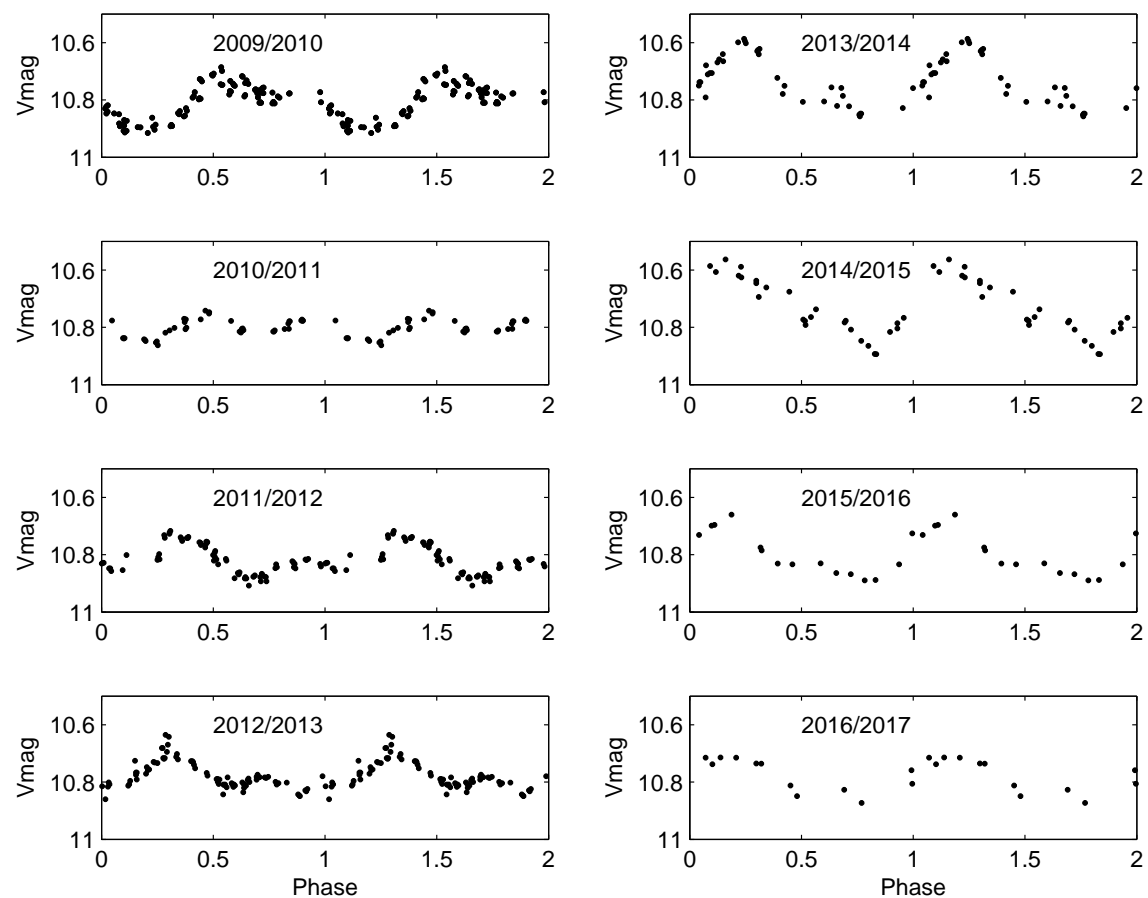

Рис. 6. Сезонные фазовые кривые блеска V410 Tau в полосе $V$, полученные в течение спектрального мониторинга 2009-2016 гг. Изменения формы и амплитуды кривой блеска обусловлены изменением как площади пятен, так и их распределением по поверхности

две области со средними фазами 0.6 и 0.75, а в 2013-2016 гг. она находится в диапазоне фаз 0.750.90. Основной фотометрический минимум блеска как раз приходится на эти фазы в 2013-2016 гг. (см. рис. 6). Фазовые кривые блеска подтверждают выводы, которые были получены в результате анализа спектрополяриметрических данных с использованием ZDI.

Таблица 2. Параметры моделей ZDI для V410 Tau

\begin{tabular}{|c|r|c|c|c|c|c|c|c|c|c|c|c|c|c|}
\hline Год & $N_{\text {obs }}$ & $\begin{array}{c}\text { пятна } \\
\text { темные }\end{array}$ & $\begin{array}{c}\text { пятна } \\
\text { яркие }\end{array}$ & $\begin{array}{c}B \\
(\Gamma \mathrm{c})\end{array}$ & $r_{\text {pol }}$ & $r_{\text {tor }}$ & $r_{d / \text { ool }}$ & $r_{s / \text { ool }}$ & $r_{d / \text { tor }}$ & $r_{s / \text { tor }}$ & $\begin{array}{c}B_{\text {dip }} \\
(\text { Гс })\end{array}$ & $\begin{array}{c}\text { наклон } \\
\text { диполя }\end{array}$ & фаза & $\begin{array}{c}R V \\
\left(\text { км } c^{-1}\right)\end{array}$ \\
\hline$(1)$ & $(2)$ & $(3)$ & $(4)$ & $(5)$ & $(6)$ & $(7)$ & $(8)$ & $(9)$ & $(10)$ & $(11)$ & $(12)$ & $(13)$ & $(14)$ & $(15)$ \\
\hline 2008 & 6 & 5.8 & 4.4 & 486 & 0.32 & 0.68 & 0.13 & 0.37 & 0.89 & 0.96 & 129 & 23 & 0.71 & 16.30 \\
2009 & 48 & 9.6 & 7.1 & 556 & 0.55 & 0.45 & 0.26 & 0.09 & 0.54 & 0.79 & 165 & 54 & 0.54 & 16.30 \\
2011 & 20 & 8.1 & 6.6 & 560 & 0.40 & 0.60 & 0.24 & 0.23 & 0.72 & 0.85 & 239 & 44 & 0.62 & 16.40 \\
2013 & 25 & 11.0 & 7.5 & 568 & 0.49 & 0.51 & 0.23 & 0.34 & 0.66 & 0.81 & 254 & 18 & 0.56 & 16.50 \\
2015 & 21 & 8.9 & 6.7 & 600 & 0.68 & 0.32 & 0.37 & 0.45 & 0.62 & 0.78 & 458 & 30 & 0.54 & 16.65 \\
2016 & 9 & 7.9 & 6.5 & 480 & 0.77 & 0.23 & 0.38 & 0.30 & 0.68 & 0.87 & 400 & 44 & 0.51 & 16.65 \\
\hline
\end{tabular}

Параметры моделей ZDI для каждого наблюдательного сезона приведены в таблице 2 . Содержимое столбцов следующее: (1) наблюдательный сезон; (2) количество спектральных данных; (3) относительные площади темных и (4) ярких пятен в процентах от видимой поверхности звезды; (5) средняя напряженность магнитного поля; (6) нормализованный вклад полоидального и (7) тороидального поля; (8) доля полоидального поля, которая является дипольной и (9) симметричной; (10) доля тороидального поля, которая является дипольной и (11) симметричной. В столбцах с 12 по 14 приведены характеристики дипольного компонента: (12) напряженность поля; (13) наклон относительно оси вращения в градусах; (14) фаза полюса диполя. В 15-м столбце дано значение системной $R V$, вычисленной с помощью ZDI.

Поскольку набор данных за декабрь 2008 г. имеет плохой фазовый охват, полученные параметры, характеризующие топологию магнитного поля в эту эпоху, имеют очень низкую достоверность и не использовались для последующего анализа и обсуждения. Из таблицы 2 видно, что в разные наблюдательные сезоны степень покрытия поверхности темными и яркими областями варьировалась 
в пределах от 15 до $18 \%$, а напряженность магнитного поля достигала значений $0.5-0.6$ кГс. Поскольку метод ZDI имеет чувствительность только к средне- и крупномасштабным поверхностным элементам и возвращает решение с учетом максимальной энтропии, такая небольшая степень покрытия пятнами не противоречит нашему предположению о том, что до 30 \% звездной поверхности может быть более или менее равномерно покрыта мелкими темными и светлыми образованиями.

Так же как яркостные карты, магнитные карты показывают сложную топологию магнитного поля с переменными полоидальным и тороидальным компонентами. Полоидальный компонент имеет слабую дипольную составляющую и является довольно неосесимметричным (см. столбцы 8 и 9 в таблице 2). Тороидальный компонент поля содержит до 45-60 \% общей магнитной энергии в 20092013 гг. и снижает свой вклад до 20-30 \% в 2015-2016 гг. В отличие от полоидального компонента, тороидальный компонент является необычно сильным и осесимметричным. Расположение полюса дипольной составляющей относительно оси вращения звезды значительно меняется от сезона к сезону в пределах от 18 до 54․ При этом фаза полюса достаточно стабильна и заключена в пределах 0.5-0.6, а интенсивность дипольного компонента постепенно нарастает с 165 Гс в январе 2009 г. до $\sim 400$ Гс в 2015-2016 гг.

\section{7 Поиск экзопланет}

После использования метода ZDI для реконструкции яркостных карт молодых звезд нашей выборки мы попытались найти признаки существования планет в спектральных данных. Значения лучевой скорости (RV), которые определяются из исходных спектров как моменты первого порядка при вычитании из континуума LSD I-профилей, содержат вклад от фотосферных неоднородностей (холодных пятен и горячих площадок), которые мы будем называть вариациями звездной активности. Восстанавливая распределение яркости по поверхности звезды с помощью метода ZDI, мы фактически выполняем фильтрацию вариаций звездной активности и получаем информацию относительно истинных значений RV звезды, и можем осуществить поиск признаков существования планеты в этих отфильтрованных значениях RV.

$\mathrm{C}$ целью поиска сигнала от планеты мы проанализировали отфильтрованные значения RV c помощью периодограммы Ломба-Скаргла для всех WTTS из нашей выборки. Периодограммный анализ проводился как для каждого сезона отдельно, так и для всего набора данных как единого временного ряда, если звезда наблюдалась в течение нескольких сезонов. В большинстве случаев мы не нашли во временных рядах отфильтрованных значений RV никаких достоверных периодов с вероятностью ложной тревоги ниже 5 \% . Тем не менее, для двух объектов, ТАР 26 и V830 Тau, нам удалось обнаружить очевидные признаки существования сигнала от планеты в отфильтрованных значениях RV.

В качестве примера на рис. 7 представлен временной ряд отфильтрованных значений RV для TAP 26 до и после извлечения сигнала от планеты. Четыре различные синусоиды на верхней панели рис. 7 показывают несколько подгонок, соответствующих различным пробным орбитальным периодам планеты: зеленая сплошная - для $P_{\text {orb }}=13.4$ сут, пурпурная сплошная - для 10.8 сут, голубая штрих-пунктирная - для 17.5 сут и черная пунктирная - для 9.1 сут. Согласно результатам периодограммного анализа, наибольшую достоверность имеет орбитальный период $P_{\text {orb }}=10.79$ сут (см. рис. 8, левая панель). Используя наиболее вероятный период и предполагая, что планета вращается в экваториальной плоскости звезды, мы получаем, что планета TAP 26b имеет минимальную массу $M \sin i=1.66 \pm 0.31 M_{J u p}$ и расположена на расстоянии $0.0968 \pm 0.0032$ а.е. от звезды.

В случае V830 Tau, мы обнаружили в наборе отфильтрованных данных RV надежный синусоидальный сигнал с полуамплитудой $68 \pm 11 \mathrm{~m} \mathrm{c}^{-1}$, который соответствует $P_{\text {orb }}=4.93$ сут (см. рис. 8 , правая панель). Используя этот период и основные параметры центральной звезды, мы получаем,

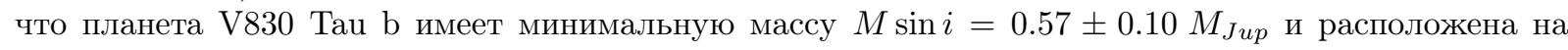
расстоянии $0.057 \pm 0.001$ a.e. от звезды.

Свойства планет, которые мы обнаружили в молодых системах V830 Таu и ТАР 26, лучше объясняются в рамках теории дисковой миграции 2-го типа, чем моделью планетного рассеивания в сочетании с приливной циркуляризацией. По сравнению с молодой WTTS V830 Таu, с возрастом в 2 млн лет, ТАР 26, с возрастом $\simeq 17$ млн лет и с аналогичной массой, выглядит как более проэволюционировавшая звезда, вращающаяся в 4 раза быстрее, чем V830 Tau, вероятно, из-за ее в 4 раза меньшего момента инерции (согласно современным моделям эволюции). Скорее всего, ее планета 


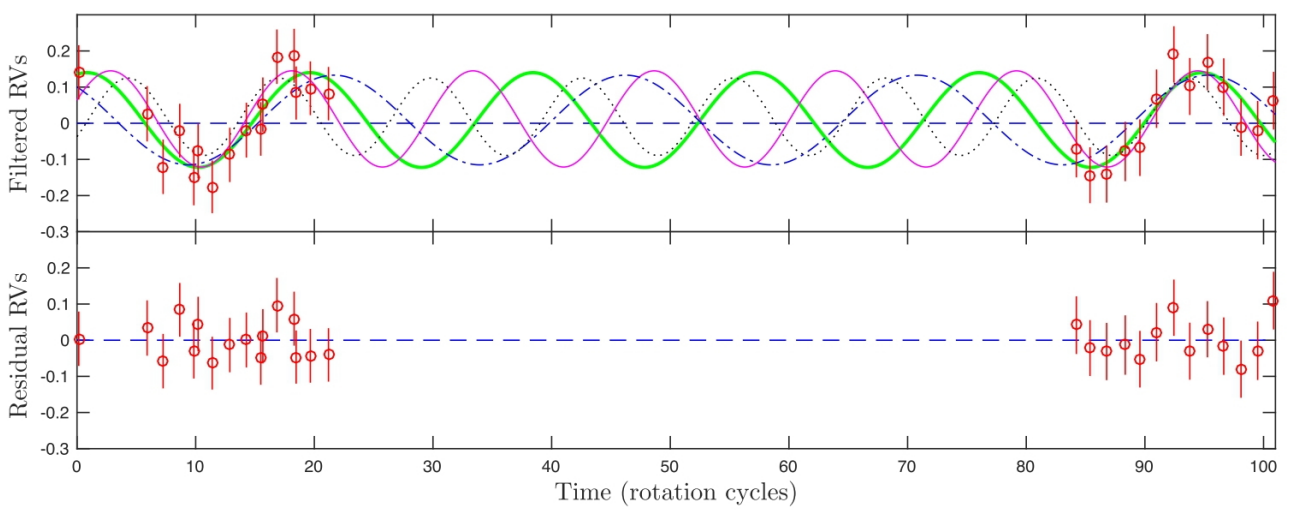

Рис. 7. Отфильтрованные значения RV для TAP 26 (верхняя панель) и остаточные RV после извлечения планетного сигнала (нижняя панель). Значения RV приведены в км с ${ }^{-1}$. Подробности в тексте (рис. 9 из работы Yu et al., 2017)
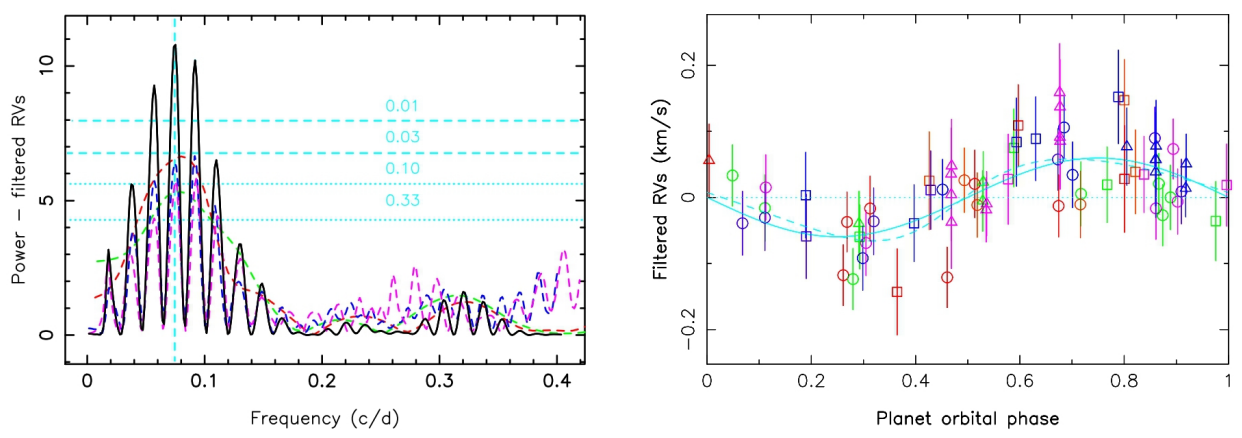

Рис. 8. Результаты периодограммного анализа данных RV для TAP 26 (слева) и фазовая кривая отфильтрованных RV V830 Таu для $P_{\text {orb }}=4.93$ сут (справа). На левой панели разным цветом представлены периодограммы, построенные для всего набора данных (черная линия), для ноября 2015 г. (красная), для января 2016 г. (зеленая), для всех нечетных данных (синяя) и для всех четных данных (пурпурная). Уровни ложной тревоги показаны горизонтальными линиями. Пик, соответствующий $P_{\text {orb }}=10.8$ сут, обозначен вертикальной штриховой линией. На правой панели разным цветом и разными значками показаны значения $\mathrm{RV}$, соответствующие различным сезонам и различным циклам вращения (рис. 10 из работы Yu et al., 2017 и рис. 8 из Donati et al., 2017)

TAP 26b закончила свою миграцию внутри диска на текущем орбитальном расстоянии (0.097 а.е.), когда ТАР 26 была еще молодой, полностью конвективной звездой с сильным дипольным компонентом магнитного поля в несколько кГс, которое могло разрушить диск на расстоянии 0.09 а.е. И это разрушение диска произошло довольно быстро, когда звезда имела возраст около 1-2 млн лет, т. е. еще до того, как крупномасштабное магнитное поле стало более слабым с более сложной топологией.

Можно предположить, что более молодая планета V830 Tau b, чей орбитальный период длиннее, чем период вращения центральной звезды, будет мигрировать до тех пор, пока звезда не замедлит свое вращение. Вопрос о том, будут ли приливные силы все еще достаточно сильными к тому времени для того, чтобы успешно переместить планету ближе к звезде и спровоцировать ее падение через несколько сотен миллионов лет, остается открытым.

\section{8 Заключение}

В результате осуществления двух международных исследовательских программ МаРP и MaTYSSE были собраны обширные фотометрические и спектрополяриметрические данные для восьми TTS в 
области звездообразования Тельца-Возничего. Анализ этих данных с помощью томографического метода ZDI позволил восстановить распределение яркости по поверхности звезд и реконструировать крупномасштабную топологию магнитного поля TTS.

Было показано, что две CTTS (AA Tau и LkCa 15) имеют осесимметричные полоидальные магнитные поля с сильным дипольным компонентом и находятся в режиме пропеллера, когда вращение молодой звезды тормозится из-за магнитной связи между звездой и протопланетным диском. Третья CTTS (V2129 Oph) демонстрирует сильное полоидальное поле, которое не является осесимметричным, причем октупольная составляющая в 2-3 раза сильнее диполя. Кроме того, было обнаружено, что магнитное поле этой звезды является нестационарным на временном масштабе в несколько лет. Изменение свойств магнитного поля выявлено и у WTTS V410 Таu. Показано, что интенсивность дипольного компонента магнитного поля монотонно увеличивалась от 165 до 458 Гс в течение 6 лет. При этом вклад дипольного компонента в полоидальное поле менялся в пределах от 23 до $38 \%$.

Анализ отфильтрованных значений RV пяти WTTS позволил впервые обнаружить признаки существования гигантских экзопланет у ТАP 26 и V830 Таu с массами порядка 1.7 и $0.6 M_{J u p}$ соответственно. Эти планеты расположены на расстояниях около 0.097 и 0.057 а.е. от TAP 26 и V830 Tau соответственно, что хорошо согласуется с теорией дисковой миграции 2-го типа.
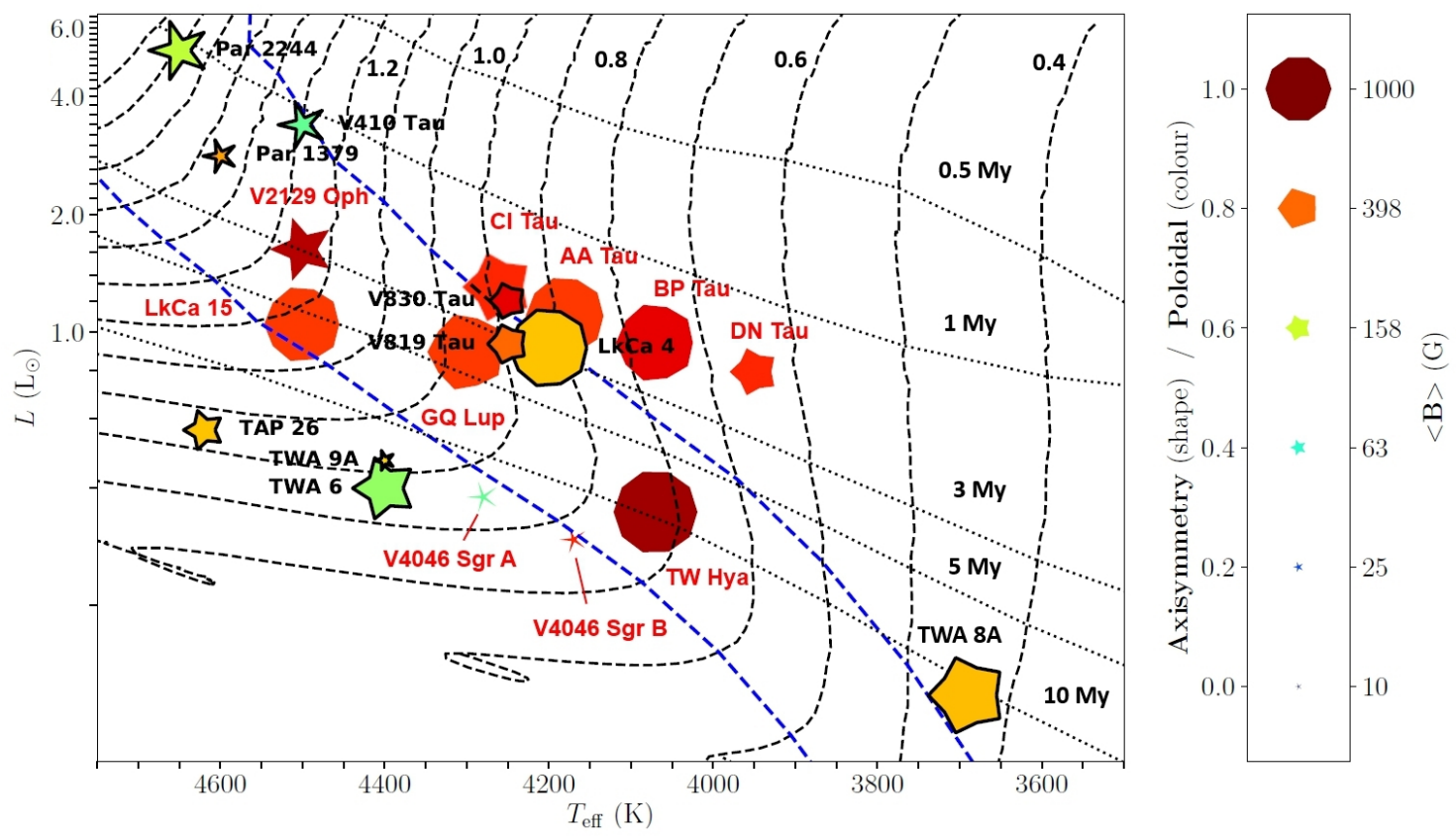

Рис. 9. ГР-диаграмма с MaTYSSE WTTS (имена выделены черным цветом) и MaPP CTTS (имена выделены красным цветом). Размер символов представляет среднюю напряженность магнитного поля. Цвет символа зависит от вклада полоидального компонента (коричневый цвет соответствует полностью полоидальному полю). Форма символа соответствует степени симметрии полоидального компонента (высокая степень симметрии представлена более округлой формой символа). Черные штриховые линии - эволюционные треки (от 0.4 до $1.9 M_{\odot}$ ), черные пунктирные линии - изохроны (для возраста 0.5, 1, 3, 5 и 10 млн лет). Две синие штриховые линии показывают долю конвективной оболочки по радиусу звезды (100 и $50 \%)$

Исследованные в нашей выборке пять WTTS демонстрируют более широкий диапазон топологий магнитного поля по сравнению с топологией магнитного поля трех CTTS. Чтобы убедиться в этих предварительных выводах, нужны данные о магнитных полях более представительной выборки молодых звезд. С этой целью мы привлекли информацию о топологии магнитного поля всех исследованных на сегодняшний день TTS, которая была доступна из недавних публикаций (см., например, Hill et al., 2019). На рис. 9 представлена ГР-диаграмма, где показаны топологии магнитного поля десяти CTTS и десяти WTTS, которые были исследованы с помощью метода ZDI в рамках программ MaPP и MaTYSSE. Данный рисунок построен на основе наших первоначальных графиков, которые были использованы в первых обоснованиях проектов МаРP и MaTYSSE в 2013-2015 гг., 
и которые впоследствии несколько раз публиковались группой МаTYSSE в разных вариантах. В частности, одна из последних версий была приведена а работе Hill et al. (2019). Настоящий рис. 9 дополнен новыми данными о топологии магнитного поля CI Таu. Для удобства чтения на рисунок нанесены имена звезд, подписаны эволюционные треки и отмечены изохроны.

Из рис. 9 видно, что в отличие от WTTS из проекта MaTYSSE, CTTS из проекта МаРP демонстрируют более сильные магнитные поля, в которых доминирует осесимметричная полоидальная компонента. Если рассматривать выборку WTTS и CTTS как единую группу TTS, то можно отметить, что магнитное поле в основном полоидальное и осесимметричное у более холодных (менее 4300 K) молодых звезд, которые остаются в значительной степени конвективными. Кроме того, более горячие (более массивные) TTS имеют менее полоидальные и менее осесимметричные магнитные поля независимо от степени развития лучистого ядра и размеров внешней конвективной оболочки (V2129 Oph, V410 Tau, Par 1379 и Par 2244). Эти выводы требуют подтверждения на основе более представительной выборки молодых звезд.

Мы подчеркиваем важность изучения крупномасштабных магнитных полей для более широкой выборки TTS с целью тестирования и развития моделей звездного динамо и теорий формирования звезд солнечного типа и их планет. Спектрополяриметры-велоситометры нового поколения, работающие в ближнем инфракрасном диапазоне, такие как SPIRou (Artigau et al., 2014), способные обеспечить доступ к еще более молодым звездам и имеющие высокую чувствительность к магнитным полям и лучевым скоростям, должны стать основным активом для успешного решения озвученных выше задач.

Благодарности. Авторы выражают благодарность референту за полезные замечания, которые позволили устранить ряд недостатков первоначального варианта статьи. Работа поддержана грантом Правительства Российской Федерации и Министерства науки и высшего образования Российской Федерации (№ 075-15-2020-780).

\section{Литература}

Alencar S.H.P, Bouvier J., Donati J.-F., Alecian E., Folsom C.P., Grankin K., et al., 2018. Astron. Astrophys., vol. 620, id. A195.

Artigau E., Kouach D., Donati J.-F., et al., 2014. Proceedings of the SPIE, vol. 9147, id. 914715.

Grankin K.N., Bouvier J., Herbst W., Melnikov S.Yu., 2008. Astron. Astrophys. vol. 479, pp. 827-843.

Grankin K.N., Artemenko S.A., 2009. Inf. Bull. Var. Stars, no. 5907, pp. 1-4.

Grankin K.N., 2013. Astron. Lett., vol. 39, no. 7, pp. 01-12.

Donati J.-F., Jardine M.M., Gregory S.G., Petit P., Bouvier J., et al., 2007. Mon. Not. Roy. Astron. Soc., vol. 380, pp. 1297-1312.

Donati J.-F., Skelly M.B., Bouvier J., Gregory S.G., Grankin K.N., et al., 2010. Mon. Not. Roy. Astron. Soc., vol. 409, pp. 1347-1361.

Donati J.-F., Bouvier J., Walter F.M., Gregory S.G., Skelly M.B., et al., 2011. Mon. Not. Roy. Astron. Soc., vol. 412, pp. 2454-2468.

Donati J.-F., Hébrard E., Hussain G., Moutou C., Grankin K., et al., 2014. Mon. Not. Roy. Astron. Soc., vol. 444, pp. 3220-3229.

Donati J.-F., Hébrard E., Hussain G.A.J., Moutou C., Malo L., Grankin K., et al., 2015. Mon. Not. Roy. Astron. Soc., vol. 453, pp. 3706-3719.

Donati J.-F., Yu L., Moutou C., Cameron A.C., Malo L., Grankin K., et al., 2017. Mon. Not. Roy. Astron. Soc., vol. 465, pp. 3343-3360.

Donati J.-F., Bouvier J., Alencar S.H., Hill C., Carmona A., Folsom C.P., et al., 2019. Mon. Not. Roy. Astron. Soc., vol. 483, pp. L1-L5.

Morin J., Donati J.-F., Petit P., Delfosse X., Forveille T., et al., 2008. Mon. Not. Roy. Astron. Soc., vol. 390, pp. 567-581.

Skelly M.B., Donati J.-F., Bouvier J., et al., 2010. Mon. Not. Roy. Astron. Soc., vol. 403, pp. 159-169.

Hill C.A., Folsom C.P., Donati J.-F., et al., 2019. Mon. Not. Roy. Astron. Soc., vol. 484, pp. 5810-5833.

Yu L., Donati J.-F., Hébrard E., Moutou C., Malo L., Grankin K., et al., 2017. Mon. Not. Roy. Astron. Soc., vol. 467, pp. 1342-1359.

Yu L., Donati J.-F., Grankin K., et al., 2019. Mon. Not. Roy. Astron. Soc., vol. 489, pp. 5556-5572. 


\title{
T Tauri stars: magnetic fields and planets
}

\author{
K. Grankin and the MaTYSSE collaboration \\ Crimean Astrophysical Observatory, Nauchny, 298409 \\ konstantin.grankin@craocrimea.ru
}

\begin{abstract}
In this short review we present the results of a study of the large-scale magnetic topologies of T Tauri stars (TTS). A small spectropolarimetric survey of 8 young stars was carried out in the framework of two international projects MaPP (Magnetic Protostars and Planets) and MaTYSSE (Magnetic Topologies of Young Stars and the Survival of massive close-in Exoplanets) between 2009 and 2016. For each of our targets we reconstructed the brightness map and the magnetic field topology using Zeeman-Doppler imaging (ZDI). This review contains a brief description of spectropolarimetric data, the ZDI method, one example of reconstruction of brightness and magnetic maps, and the properties of magnetic fields of 8 TTS. Our results suggest that AA Tau and LkCa 15 interact with their discs in a propeller mode, when their rotation is actively slowed by the star/disc magnetic coupling. We find that magnetic fields of some TTS are variable on a time scale of a few years and are thus intrinsically non-stationary. We report the detection of a giant exoplanet around V830 Tau and TAP 26. These two new detections suggest that disc type II migration is efficient at generating newborn hot Jupiters (hJs) around young TTS. The result of our survey is compared to the global picture of magnetic field properties of twenty TTS in the Hertzsprung-Russell diagram. The comparison shows that WTTS exhibit a wider range of field topologies compared to CTTS, and all TTS (CTTS and WTTS as a whole) are mostly poloidal and axisymmetric when they are mostly convective and cooler than $4300 \mathrm{~K}$. This needs to be confirmed with a larger sample of stars.
\end{abstract}

Key words: young stars, magnetic fields, protoplanetary disks, exoplanets 